\title{
Transmission Electron Microscopy and Luminescence Studies of Quantum Well Structures Resulting from Stacking Fault Formation in $4 \mathrm{H}-\mathrm{SiC}$ Layers
}

\author{
J. Borysiuk ${ }^{a, b, *}$, A. WysmołeK ${ }^{b}$, R. BożeK ${ }^{b}$, W. Strupiński ${ }^{a}$ \\ AND J.M. BARANOWSKI ${ }^{b}$ \\ ${ }^{a}$ Institute of Electronic Materials Technology \\ Wólczyńska 133, 01-919 Warsaw, Poland \\ ${ }^{b}$ Institute of Experimental Physics, University of Warsaw \\ Hoża 69, 00-681 Warsaw, Poland

\begin{abstract}
Transmission electron microscopy and photoluminescence studies of quantum well structures related to stacking faults formation in $4 \mathrm{H}-\mathrm{SiC}$ homoepitaxial layers are reported. The investigated $4 \mathrm{H}-\mathrm{SiC}$ layers were deposited on $8^{\circ}$ misoriented Si-terminated (0001) surface of high quality $4 \mathrm{H}-\mathrm{SiC}$ substrate. It is found that the planar defects created by direct continuation from the $\mathrm{SiC}$ substrates are cubic 3C-SiC stacking faults. These defects are optically active, giving rise to characteristic luminescence band in the spectral range around $2.9 \mathrm{eV}$, which consist of several emission lines. The observed energy and intensity pattern of this emission is discussed of in terms of single, double and multiple quantum wells formed from neighboring 3C-SiC SF layers embedded in $4 \mathrm{H}-\mathrm{SiC}$ material.
\end{abstract}

PACS numbers: 68.37.Lp, 78.55.-m, 61.72.Nn, 68.65.Fg, 61.72.up

\section{Introduction}

Hexagonal silicon carbide including $4 \mathrm{H}-\mathrm{SiC}$ is very promising for high-power semiconductor devices [1]. However, the full scale commercialization of hexagonal $\mathrm{SiC}$ is still hampered by structural instability issues mostly caused by spontaneous stacking faults (SF) formation [1-3]. On the other hand, low formation energy of 3C-SiC stacking fault inclusions in higher band-gap $\mathrm{SiC}$ polytypes provides a unique possibility to obtain quantum well (QW) structures without changing of chemical composition of epitaxial layers during growth [4]. Such a concept of "structure-only" QW would be interesting from the point of view of applica-

*corresponding author; e-mail: jolanta.borysiuk@itme.edu.pl 
tions, provided precise control of $\mathrm{SF}$ formation during the growth of $\mathrm{SiC}$ based nanostructures as well as possibility of tailoring of their electronic properties is attainable.

In this report transmission electron microscopy (TEM) and low temperature photoluminescence studies of single and multiple $3 \mathrm{C}-\mathrm{SiC}$ stacking faults formed in $4 \mathrm{H}-\mathrm{SiC}$ homoepitaxial layers are presented.

\section{Experimental}

High quality $4 \mathrm{H}-\mathrm{SiC}$ substrates were used for the deposition of lattice matched silicon carbide layer. The substrates were misoriented by $8^{\circ}$ to basal (0001) silicon-terminated plane, along [11 20$]$ direction. SiC epitaxial layer (about $10 \mu \mathrm{m}$ thick) was deposited in Epigress VP508 hot wall chemical vapor deposition (CVD) reactor, using $\mathrm{SiH}_{4}$ and $\mathrm{C}_{3} \mathrm{H}_{8}$ sources of silicon and carbon, respectively. The temperature of the growth was $1350^{\circ} \mathrm{C}$.

TEM observations were performed on a JEOL JEM 3010 electron microscope operating at an accelerating voltage of $300 \mathrm{kV}$. Cross-sectional TEM specimens were prepared by a standard method of mechanical pre-thinning followed by $\mathrm{Ar}$ ion milling. Photoluminescence (PL) measurements were performed at $4.2 \mathrm{~K}$ using the $325 \mathrm{~nm}$ line from $\mathrm{He}-\mathrm{Cd}$ laser as a source of continuous wave excitation. Laser spot size on the sample surface was about $1-2 \mu \mathrm{m}$.

\section{TEM and PL results}

TEM and PL measurements confirmed high crystallographic quality of the investigated layers. No distinct interface between the substrate and the layer was detected. No signatures of lattice mismatch related strain between the layer and the substrate was found.

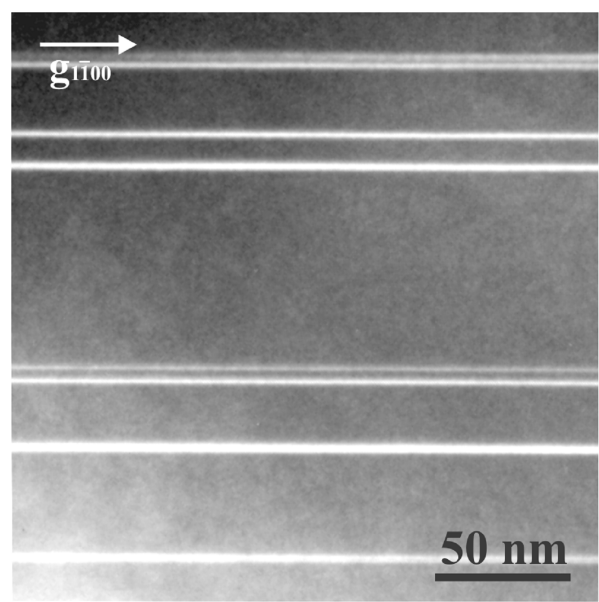

Fig. 1. TEM dark field image of the $4 \mathrm{H}-\mathrm{SiC}$ epitaxial layer. Randomly spaced SF doublets are clearly visible. 

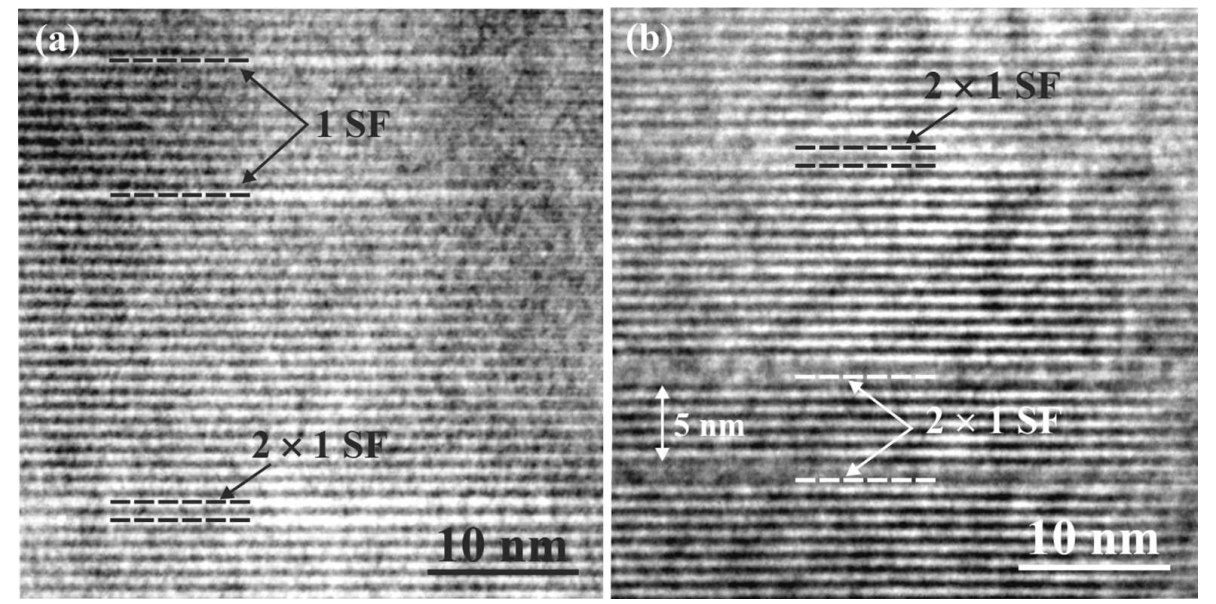

Fig. 2. HRTEM images of single stacking fault (1SF) (a) and double stacking fault structure $(2 \times 1 \mathrm{SF})(\mathrm{b})$.

The $4 \mathrm{H}-\mathrm{SiC}$ substrates and epitaxially grown layers were investigated using transmission electron microscopy. Typical TEM dark field image of the investigated $4 \mathrm{H}-\mathrm{SiC}$ epitaxial layers is presented in Fig. 1. It is observed that over the entire investigated sample area randomly distributed single stacking faults and stacking fault doublets are present. They arise as a direct continuation of SF existing in the substrate. The structure of the stacking faults was analyzed using high resolution transmission electron microscopy (HRTEM). Representative HRTEM image of the investigated layer is presented in Fig. 2. Both single SF $(1 \mathrm{SF})$ and double $(2 \times 1 \mathrm{SF})$ stacking faults are shown. The atomic sequence corresponds to cubic arrangement of atomic layers, i.e. we observe cubic planar defect inclusions (3C-SiC) within hexagonal $4 \mathrm{H}-\mathrm{SiC}$ matrix. The displacement vector was identified to be: $R= \pm(1 / 3)[1 \overline{1} 00]$ or $R= \pm(1 / 3)[10 \overline{1} 0]$ for the analyzed SFs. Thus the observed SF are single type Shockley faults. It is observed that distances between neighboring SF may vary from 1 to $5 \mathrm{~nm}$.

A typical low-temperature micro-luminescence spectrum of the investigated $4 \mathrm{H}-\mathrm{SiC}$ layer measured in the broad energy range is presented in Fig. 3. In addition to the $P$ and $Q$ excitonic lines characteristic of high quality $4 \mathrm{H}-\mathrm{SiC}$ doped with nitrogen, a group of new emission lines appears around energy of $2.9 \mathrm{eV}$. In contrary to the emission due to nitrogen-bound excitons, which remains stable with changing position on the sample (see Fig. 4a), the energy as well as the intensity pattern related to SF related luminescence show periodic like changes with scanning on the sample surface (see Fig. 4b).

Similar spectra observed in this spectral region in the highly $n$-doped $4 \mathrm{H}$ $\mathrm{SiC}$ crystals [3] as well as for $4 \mathrm{H}-\mathrm{SiC} p-n$ diodes after extended operation [5] were attributed to the interband transitions involving electrons localized in QWs induced by single $3 \mathrm{C}-\mathrm{SiC}$ stacking faults. In order to explain the number of the 


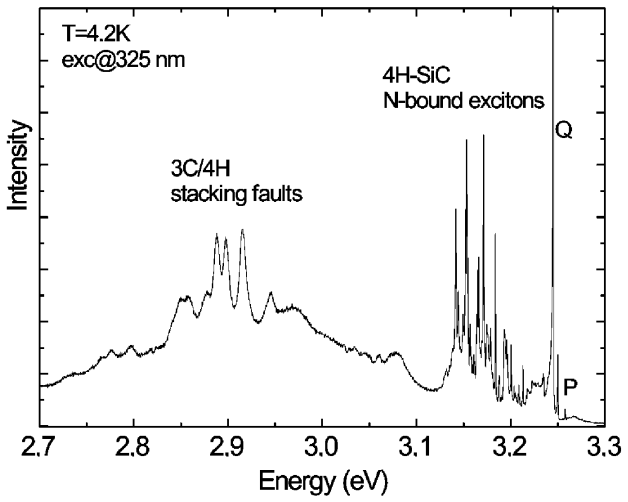

Fig. 3. Low temperature PL spectrum of $4 \mathrm{H}-\mathrm{SiC}$ layer measured in the broad energy range.

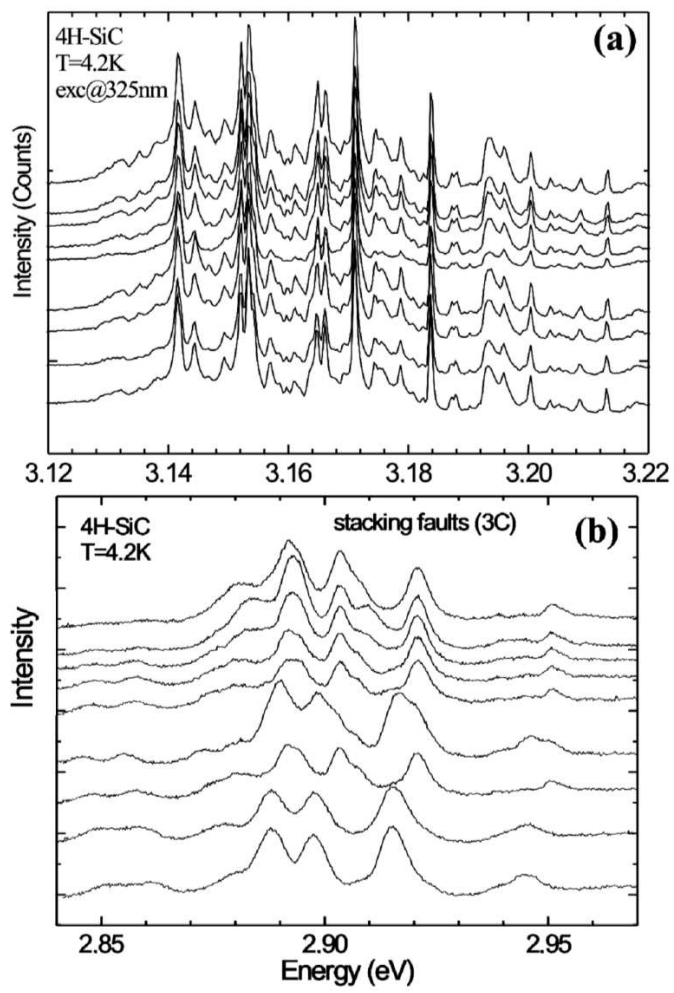

Fig. 4. The photoluminescence spectra measured for different positions on the $4 \mathrm{H}-\mathrm{SiC}$ layer for the nitrogen-bound exciton recombination (a), and in the spectral region around $2.9 \mathrm{eV}$, corresponding to the $\mathrm{SF}$ induced recombination (b).

observed lines as well as energy positions of the particular transitions constituting the $2.9 \mathrm{eV}$ band, we propose that in addition to the emission due to $3 \mathrm{C}-\mathrm{SiC}$ single SF, the luminescence arising at randomly separated SF doublets, forming 


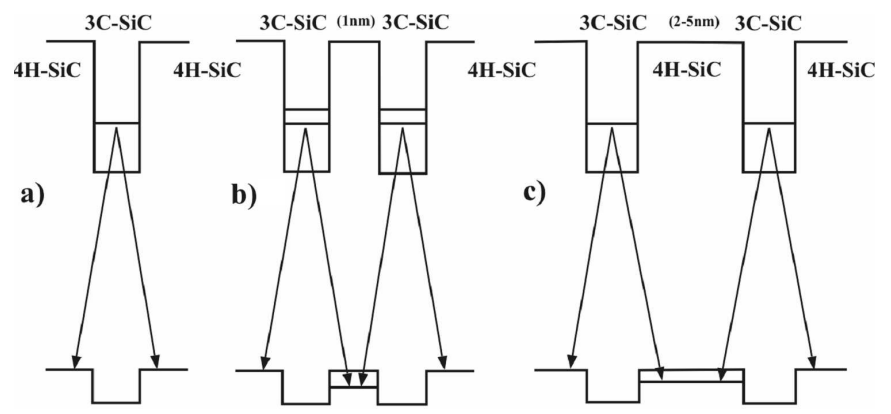

Fig. 5. Schematic band diagrams showing potential arrangements for electron and holes in QW structures, induced by (a) single 3C-SiC stacking fault, (b) double-SF separated by $1 \mathrm{~nm}$, and (c) double-SF separated by $2-5 \mathrm{~nm}$ distance. Some possible optical transitions are marked by arrows.

type II QW structures are involved (see Fig. 5). According to the recombination scheme presented in Fig. 5, the energies of the transitions involving neighboring QW levels should strongly depend on the SFs separation. As shown by TEM studies, the distances between neighboring SF are restricted to the well defined values corresponding of the multiples of atomic layers in $4 \mathrm{H}-\mathrm{SiC}$. Thus, it would be expected that in the emission spectrum of the SF-doublets only transitions at some distinct energies should be present. This seems to be observed experimentally. The energy of emission lines attributed to SF shows quasi-periodic jumps between well defined energies. The observed intensity pattern changes in a similar way (see Fig. 4b). In order to confirm the presented model of the SF induced emission in $4 \mathrm{H}-\mathrm{SiC}$ layers additional experiments as well theoretical efforts are needed.

\section{Summary}

TEM and PL studies showed that the $3 \mathrm{C}-\mathrm{SiC}$ stacking faults formed in $4 \mathrm{H}-\mathrm{SiC}$ layers grown on the $8^{\circ}$ misoriented $4 \mathrm{H}-\mathrm{SiC}$ substrates are direct continuation from the substrate. The stacking faults create a new type of "structure-only" quantum wells. It is proposed that closely neighboring stacking faults form type-II quantum well structures responsible for the $2.9 \mathrm{eV}$ emission band.

\section{Acknowledgments}

The research reported in this study was partially supported by Ministry of Science and Higher Education grants N 507138 32/4046, PBZ-6/2/2006 and MTKD-CT-2005-029671 EU program. The authors thank the Faculty of Materials Science and Engineering of Warsaw University of Technology for permission to use transmission electron microscope JEOL JEM3010. 


\section{References}

[1] M. Skowronski, S. Ha, J. Appl. Phys. 99, 011101 (2006).

[2] D. Nakamura, I. Gunjishima, S. Yamaguchi, T. Ito, A. Okamoto, H. Kondo, S. Onda, K. Takatori, Nature (London) 430, 1009 (2004).

[3] A. Galeckas, A. Hallen, S. Majdi, J. Linnros, Phys. Rev. B 74, 233203 (2006) and references therein.

[4] Y. Ding, K.-B. Park, J.P. Pelz, K.C. Palle, M.K. Mikhov, B.J. Skromme, H. Meidia, S. Mahajan, Phys. Rev B 69, 041305(R) (2004).

[5] A.G. Srighara, F.H.C. Carlsson, J.P. Bergman, E. Janzén, Appl. Phys. Lett. 79, 3944 (2001). 